\title{
Tecnologías de la Información y la Comunicación (TIC) como Estrategia para Prevenir la Contaminación Ambiental ${ }^{1}$
}

\section{Information and communication technologies (TIC) as a strategy to prevent environmental pollution}

DOI: http://dx.doi.org/10.17981/cultedusoc.9.3.2018.83

Artículo de investigación. Fecha de recepción: 15/06/2018. Fecha de aceptación: 27/11/2018

\author{
Daisy Munive Ruiz², \\ Carmen Acosta Altamar; Rosa Bermúdez Cantillo y Alejandra Ramos Guevara ${ }^{3}$ \\ Institución San Martín de Porres (Colombia) \\ institucionsanmartindeporres@gmail.com
}

Para citar este artículo:

Munive, D., Acosta, C., Bermúdez, R. y Ramos, A. (2018). Tecnologías de la Información y la Comunicación (TIC) como Estrategia para Prevenir la Contaminación Ambiental. Cultura. Educación y Sociedad 9(3), 709-714. DOI: http://dx.doi.org/10.17981/cultedusoc.9.3.2018.83

\section{Resumen}

El uso de las Tecnologías de la Información y la Comunicación (TIC), se ha expandido a nivel mundial y por lo tanto se encuentra presente en cada una de los ámbitos en los que se desempeña el ser humano. Por tal motivo, se consideró la estrategia a emplear en este proyecto, con el objetivo de mitigar y prevenir la contaminación ambiental con ayuda de los estudiantes de la Institución Educativa Departamental (IED) San Martín de Porres y de la Comunidad de Fundación, Magdalena. La metodología utilizada fue un estudio cualitativo y descriptivo, utilizando el modelo de investigación acción-participativa entre estudiantes, la comunidad y los docentes. Para recoger los datos, se utilizaron relatorías individuales, institucionales y el diario de campo. Se evidenció durante el desarrollo del proyecto, una problemática de malas prácticas en los criaderos que se encuentran alrededor de la institución, lo cual ocasiona contaminación ambiental y daños en la salud de las personas.

Palabras clave: Tecnología de la Información y la Comunicación, estrategia pedagógica, prevención de la contaminación ambiental, educación básica.

\section{Abstract}

The use of Information and Communication Technologies (ICT) has expanded worldwide and therefore is present in each of the areas in which the human being performs. For this reason, they were considered as the strategy to be used in this project, with the aim of mitigating and preventing environmental contamination with the help of the IED students San Martín de Porres and the community of Fundación, Magdalena. The methodology used was a qualitative and descriptive study, using the action-participatory research model among students, community and teachers. In order to collect the data, individual, institutional and field diary reports were used. During the development of the project, a problem of bad practices was shown in the breeding sites that are located around the institution, which causes environmental contamination and damage to the health of the people.

Keywords: Information and Communication Technology, pedagogical strategy, prevention of environmental pollution, basic education. 1 Este artículo ha sido derivado del Programa de Fortalecimiento de la Cultura Ciudadana y Democrática CT+I a través de la IEP
apoyada en TIC en el Departamento de Magdalena: CICLON

2 Líder del grupo de investigación “Las Amigas De La Investigación”.

3 Docentes de la Institución San Martín de Porres.

- The author; licensee Universidad de la Costa - CUC. 


\section{Introducción}

La tecnología de la información y comunicación (TIC), ha repercutido directamente en la vida cotidiana, en el entorno social, en todos los campos de la sociedad, y en este sentido, la escuela como institución social, no escapa a esa influencia, ya que las TIC en este ámbito actúan como herramienta para fortalecer el desarrollo de la educación (Abraham y Vitarelli, 2014). En este sentido, el uso de las TIC, brindan a las personas que se quieran educar una nueva forma de hacerlo, modificando así sus hábitos y la perspectiva de lo que aprende (Corredor y Sandino 2009), y ofrecen posibilidades para el desarrollo de trabajos colaborativos, potenciando la mirada ambiental desde la perspectiva del análisis espacial a diferentes escalas, ya que los problemas ambientales no sólo remiten a lo local, sino que, tal como lo señala Cruz (2016), pueden referenciarse en contextos más amplios y complejos, con la posibilidad de contar con información y puntos de vista multiculturales (Ariza, Meza y Meza, 2018), así como de comprender fenómenos desde el aporte de personas que se encuentran trabajando en otros espacios y tiempos (Abraham y Vitarelli, 2014).

Por ello, en esta última década ha sido de vital importancia la generación de competencias TIC por parte del sistema educativo , estructurada desde una perspectiva de índole transversal en los procesos de enseñanza-aprendizaje y el ejercicio profesional sea adecuado a las nuevas demandas laborales (Chumaceiro, Hernández y otras; 2017).

Por otra parte, durante los últimos años Colombia se ha dado a la tarea de cuantificar el impacto asociado a la contaminación ambiental e identificar los principales contribuyentes a este fenómeno (Valencia et al., 2010). En Colombia, para lograr un uso eficiente de los recursos ambientales en especial los recursos hídrico, el ministerio del medio ambiente, en el año 2010, propuso la política para la gestión integral del recurso hídrico, con la cual se busca la regulación de las diferentes etapas de la utilización del agua; teniendo en cuenta la importancia de este elemento para nuestra vida (Salamanca, 2016).
Debido a esto, en el Departamento del Magdalena se evidenció mediante un estudio ambiental realizado en 18 municipios, entre ellos Fundación, que las problemáticas ambientales principales son: la generación de residuos sólidos, la disminución y contaminación del recurso hídrico, la deforestación, el mal uso y sedimentación de cuerpos de agua y, extinción de fauna silvestre (Díaz, 2016).

Por consiguiente, las áreas aledañas al matadero público y la agroindustria (queseras y extractoras) de Fundación, son consideradas focos de alta contaminación, por los vertimientos de las aguas residuales a los lechos del Río, caños y vías sin ningún tipo de tratamiento (Álvarez, 2000).

Por tal motivo, atendiendo las necesidades específicas ambientales y de salud en los alrededores de la escuela San Martín de Porres, del municipio de Fundación Magdalena, donde existen agentes contaminantes provenientes de porquerizas rudimentarias, un grupo conformado por cuatro docentes de esta institución, entre la jornada de la mañana y la tarde, decidió desarrollar un proyecto de línea ambiental en el marco del Macro-proyecto del Ciclón de la Universidad de la Costa. En este proyecto evidenciaron que las porquerizas rudimentarias a escasos 20 metros y otras un poco más alejadas de la IED, las cuales expiden malos olores que causan malestares a los estudiantes (dolor de cabeza, vómitos, mareos y problemas respiratorios), docentes y a la comunidad cercana a la escuela. Esta situación podría generar no sólo enfermedades como las mencionadas anteriormente, sino que a largo plazo podría causar enfermedades pulmonares por respirar bajo estas condiciones durante largos lapsos de tiempo. La influencia de los factores ambientales en la salud, en general, y en la afección respiratoria, en particular, está cada vez más clara. Si tenemos en cuenta que toda la población está expuesta a estos factores de forma constante, aun efectos de pequeña intensidad generarán consecuencias con importante repercusión en la salud de la población (González, Rodríguez, Cruz y Martínez 2009).

El objetivo del proyecto en su fase inicial condujo a identificar bajo la propuesta de in- 
vestigación como estrategia pedagógica y las TIC, los factores que aportan en la prevención de la contaminación ambiental, involucrando a nuestros alumnos como investigadores y capacitarlos en la implementación y uso de las TIC como herramienta para contribuir a resolver futuros problemas.

\section{Metodología}

Como parte de los aspectos metodológicos, la investigación contempló técnicas de recolección de información e instrumentos, así como las fuentes de información primaria (la comunidad), a partir de entrevistas, narraciones, grabaciones, transcripciones de audio, vídeo, fotografías o películas (Meza et al. 2018; Pérez, 2002; Hernández, 2014). La investigación se llevó a cabo mediante un diseño descriptivo, en donde se definieron los espacios (salidas de campo) (Hidalgo, Cordero, Sandí y Cruz, 2015), para llevar a cabo las observaciones en los alrededores de la IED San Martín de Porres.

\section{Recursos: TIC más Utilizadas}

Como herramienta pedagógica para la toma, análisis y feedback de la información, se utilizaron las TIC principales evidenciadas en la investigación de Cabero y Llorente (2005), donde se determinaron que son las siguientes:

- El video.

- La informática.

- Multimedia e internet.

\section{Métodos: Tecnologías de la Información y la} Comunicación (TIC)

Como metodología de la investigación se utilizaron las TIC como herramientas pedagógicas en relación a la problemática ambiental estudiada. Las funciones que se han propuesto que pueden cumplir las TIC son diversas y han sido analizadas específicamente por diferentes autores.

En la tabla 1 se pueden observar algunas de las realizadas por distintos, sabiamente recogidas por Cabero y Llorente (2005):

TABLA 1

Funciones Asignadas a las TIC.

\begin{tabular}{|c|c|c|c|c|c|c|}
\hline & $\begin{array}{l}\text { (Bullande, } \\
\text { 1969) }\end{array}$ & $\begin{array}{l}\text { (Nerici, } \\
\text { 1973) }\end{array}$ & $\begin{array}{l}\text { (Zabalza, } \\
\text { 1987) }\end{array}$ & $\begin{array}{l}\text { (Rowntree, } \\
\text { 1991) }\end{array}$ & $\begin{array}{l}\text { (Cebrián, } \\
\text { 1992) }\end{array}$ & $\begin{array}{l}\text { (Parcerisa, } \\
\text { 1996) }\end{array}$ \\
\hline Facilitadores de desarrollo profesional & & & & & * & \\
\hline Motivar/atraer la atención & * & * & * & * & & * \\
\hline Portadores de contenidos & * & * & & & * & * \\
\hline $\begin{array}{l}\text { Estructuradores de la realidad/ } \\
\text { contenidos }\end{array}$ & & * & & & * & * \\
\hline Facilita el recuerdo de la información & & * & & * & & * \\
\hline Estimular nuevos aprendizajes & & & & * & & \\
\hline $\begin{array}{l}\text { Facilitar respuestas activas por parte } \\
\text { del estudiante }\end{array}$ & & * & & * & & \\
\hline Ofrecer Feedback & & & & * & & \\
\hline Innovadora & & & * & & * & * \\
\hline Nuevas relaciones profesores-alumnos & & & * & & & \\
\hline Formativa global & & & * & & & \\
\hline Sustitutos de la realidad & * & & & & & \\
\hline Sustitución del profesor & * & & & & & * \\
\hline $\begin{array}{l}\text { Ejemplificadores de modelos de } \\
\text { enseñanza }\end{array}$ & & & & & * & \\
\hline $\begin{array}{l}\text { Facilitan un mejor análisis e } \\
\text { interpretación del tema }\end{array}$ & & * & & & & \\
\hline
\end{tabular}

Fuente: Cabero y Llorente (2005). 
Llevar a la práctica este modo de abordaje multidisciplinario conllevó indefectiblemente a propiciar el trabajo en conjunto entre los docentes, por lo tanto, el trabajo colectivo supuso "la corresponsabilidad sobre la propuesta escolar y la trayectoria de los estudiantes. Posibilitó la renovación permanente de la tarea, en la medida que permitió producir saberes sobre la enseñanza y la escolaridad (Abraham y Vitarelli, 2014).

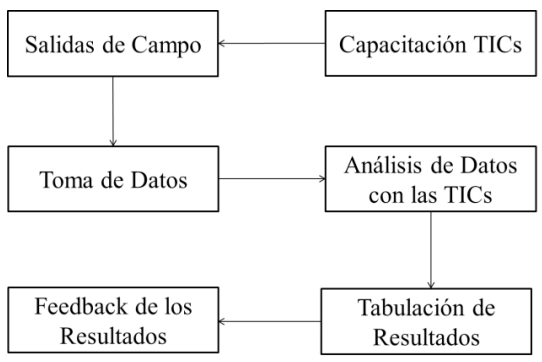

Figura 1. Ruta Metodológica General de Investigación. Trayectoria Metodológica.

Fuente: elaboración propia.

Para la recolección de información, se utilizaron las bitácoras o diarios de campo, relatorías individuales, institucionales, observación directa, encuestas, información suministrada por estudiantes y comunidad (fig. 1).

El proceso metodológico se dividió en:

- Etapa 1: Capacitación en las TIC a los alumnos participantes y acompañantes del proyecto investigativo.

- Etapa 2: Recolección y análisis de la información obtenida a través de las salidas de campo en los alrededores de la IED San Martín de Porres.

\section{Resultados}

De acuerdo con las encuestas realizadas a los docentes de la IED San Martín de Porres de la primera etapa, se tiene como principales resultados de la fig. 2 .

El 82\% de los niños encuestados respondió que sí han recibido capacitación acerca de la implementación y uso de las TIC como herramienta pedagógica para el análisis y solución de problemas de investigación, un $14 \%$ no ha recibido dichas capacitaciones debido al ausentismo por parte de algunos estudiantes por diferentes condiciones tales como (fig. 3):

- Condiciones ambientales.

- Problemas familiares.

- Enfermedades/otros.

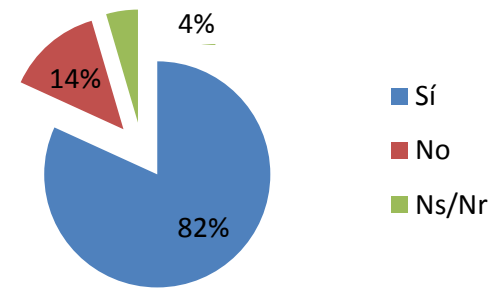

Figura 2. Capacitación al Grupo de Investigación en las TIC.

Fuente: Elaboración propia.

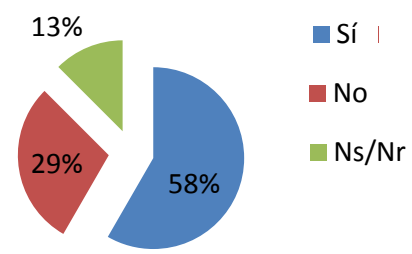

Figura 3. Manejo e Implantación de las TIC.

Fuente: Elaboración propia.

Referente al manejo y uso de las TIC, sólo el 58\% de los encuestados saben utilizar la herramienta para resolver y analizar problemas. Se evidenció que el 29\% restante si conoce la metodología pero no se les hace fácil la implementación en el campo.

\section{Resultados}

Se tabularon los datos obtenidos de las porquerizas ubicadas en los alrededores de la IED San Martín de Porres, y los resultados se muestran a continuación en la tabla 2 :

TABLA 2

Criaderos de Cerdos y Gallinas

\begin{tabular}{llll}
\hline \multicolumn{4}{c}{ Criaderos de Cerdos } \\
\hline $\begin{array}{l}\text { Tipo de } \\
\text { Animal }\end{array}$ & Cantidad & Condiciones & $\begin{array}{l}\text { Infra- } \\
\text { estructura }\end{array}$ \\
Cerdo & 14 & Mala & Inadecuada \\
Gallinas & 2 & Mala & Inadecuada \\
\hline
\end{tabular}

Fuente: elaboración propia. 
Dando respuesta al objetivo de la investigación, se identificaron los siguientes factores que contribuyen a la contaminación ambiental por parte de la comunidad:

- Existen criaderos de cerdos para la venta en casas cercanas a la institución educativa, los cuales se mantienen en malas condiciones generando malos olores en el ambiente. Estos criaderos no cuentan con la infraestructura adecuada para el manejo de estos animales, sus aguas residuales infectan en general a todo el barrio.

- Las personas que mantienen este tipo de actividad para su sustento no tienen ayuda por parte de los entes encargados de salubridad, por lo cual se hace necesario la presencia de estos en la dirección de esa labor.

- Se hace necesario fomentar el trabajo en equipo para concientizar a la población sobre la importancia de no contaminar el ambiente.

- Para los docentes se hace necesario seguir implementando la IEP en el aula de clases para que los estudiantes puedan continuar en este proyecto investigativo y contribuyan al cuidado del medio ambiente.

- Dentro del proyecto de investigación debe tenerse en cuenta un punto de educación ambiental para la comunidad, de esa manera se logrará mayor apoyo por parte de estos.

\section{Discusión}

Se logró identificar los distintos factores que se encuentran influyendo a la contaminación del aire en la zona donde se encuentra ubicada la escuela San Martin de Porres del municipio de Fundación, Magdalena. Lo anterior permite tomar las medidas necesarias para evitar que se continúe con la contaminación ambiental que hay hasta el momento.

Se hace necesaria la continuidad de este proyecto para lograr un impacto significativo en la sociedad, teniendo como aliados a las distintas entidades a quienes compete el tema de salud, educación, y específicamente el manejo de actividades relacionadas con los porcinos, ya que, si bien se está trabajando esto de manera irregular, cabe tener en cuenta que estas actividades son el sustento de varias familias de escasos recursos. Se considera importante la formación a estas familias en materia del trato a este tipo de actividades, para que mejoren el proceso, disminuya la contaminación ambiental y por ende mejoren su calidad de vida.

\section{Recomendaciones}

Teniendo en cuenta la reglamentación del Instituto Colombiano Agropecuario (ICA), basada en la Resolución 2640 (2007), los predios y granjas de producción primaria de porcinos destinados al consumo humano y con el fin de proteger la vida humana, la salud humana y el ambiente se deben tener en cuenta los siguientes requisitos sanitarios:

- Todo predio debe estar inscrito ante el ICA.

- El responsable del predio de informar al ICA todos los ingresos y salidas de los animales.

- El ingreso de animales debe reportarlo en un plazo no mayor a 30 días.

- Los predios/granjas, se deben ubicar de acuerdo al Plan de Ordenamiento Territorial POT de cada municipio.

- Deben estar alejados de fuentes de contaminación como basureros y rellenos sanitarios, estar claramente delimitados y que las cercas estén en buen estado.

- Queda prohibido tener los cerdos libres.

- Se deben proteger y mantener las fuentes de agua y realizar monitoreo constante de la calidad del agua para consumo humano.

- Todo predio debe contar con un programa documentado de control de plagas y roedores (ICA 2007, p. 1-20),

Teniendo en cuenta estas recomendaciones por parte de los predios/granjas, se espera mitigar el impacto ambiental negativo ocasionado en los alrededores de la IED San Martín de Porres y mejorar la salud de todo el cuerpo docente y estudiantil, y en general, a la comunidad cercana a los senderos de los criaderos. 


\section{Referencias}

Abraham, R. y Vitarelli, M. (2014). La Enseñanza del Ambiente y las TIC en Proyectos Educativos del Nivel Secundario en San Luis. En, Congreso Iberoamericano de Ciencia Tecnología, Innovación y Educación. (6). Buenos Aires, Argentina.

Alvarez, R. (2000). Plan Básico de Ordenamiento Territorial (PBO) 2000-2009. En, Municipio de Fundación, Plan Estructurante Territorial Fundación Siglo XXI, (20-24). Fundación, Magdalena, Colombia.

Cabero, J. y Llorente Cejudo, M. (2005). Las TIC y la Educación Ambiental. Revista Latinoamericana de Tecnología Educativa, 4, 13-14.

Chumaceiro, A., Hernández, J., Melendez, Y. y Acurero M. (2017). Responsabilidad universitaria y sociedad del conocimiento para la generación de competencias TIC. En, Procesos formativos para el siglo XXI. Cabimas: Fondounermb.

Corredor, A. y Sandino, M. (2009). Las TIC'S como Herramienta de Investigación Científica. Gondola, 4(1). 1-5.

Cruz, E. (2016). El ciclo de protesta 2010-2016 en Colombia. Una explicación. Jurídicas CUC, 12(1), 31-62. DOI: http://dx.doi.org/10.17981/ juridcuc.12.1.2016.3

Díaz, C. (2016). Informe de Gestión: Corporación Autónoma Regional del Magdalena. Magdalena: Corpamag .

González, J., Rodríguez, J., Cruz, M. y Martínez, C. (2009). Exposisción a Contaminantes Ambientales y Enfermedad Respiratoria: $U n$ Foco de Atención en el Año 2009. Archivos de Bronconeumología, 46(1). 17-20. https://doi. org/10.1016/S0300-2896(10)70005-3

Hernández, R., Fernández, C. y Batipsta, P. (2014). Metodología de la Investigación. México, D.F.: McGrawHill.

Hidalgo, K., Cordero, O., Sandí, J. y Cruz, M. (2015). Las TIC como herramienta Pedagógica en Procesos de Investigación Educativa. En, Universidad Pontificia Bolivariana, XIV Jornadas II Congreso Internacional. Investigar en la Educación y Educar en Investigación. Avances y Perspectivas, (10-11). Medellín, Colombia.
ICA (28 de septiembre de 2007). Por la cual se reglamentan las condiciones sanitarias y de inocuidad en la producción primaria de ganado porcino destinado al sacrificio para consumo humano. [Resolución 002640]. DO: 46.768 .

Meza, A., Arrieta, M. y Meza, S. (2018). Análisis de la conciliación extrajudicial civil en la Costa Atlántica colombiana. Jurídicas CUC, 14(1). 187-210. $\quad$ DOI: http://dx.doi. org/10.17981/juridcuc.14.1.2018.9

Nieves, J. y Salazar, F. (2016). e-Supply chain management para una empresa de tecnología. International Journal of Management Sciences and Operations Research, 1(1), 3134. Recuperado de http://ijmsoridi.com/index. php/ijmsor/article/view/74

Pérez, G. (2002). Desafíos de la Investigación Cualitativa. ResearchGate, 3-4.

Sagastume, A. y Cabello, J. (2017). La educación superior y una producción más limpia. International Journal of Management Sciences and Operations Research, 2(1), 4-8. Recuperado de http://ijmsoridi.com/index.php/ijmsor/article/view/79

Salamanca, E. (2016). Tratamiento de aguas para el consumo humano. Modulo Arquitectura CUC, 17(1). 29-48

Sepúlveda, A., Martínez, R., Medina, S. y Salazar, F. (2016). Propuesta de diseño de una red supply chain para la agrocadena de cacao, municipio de Viotá, Cundinamarca. International Journal of Management Sciences and Operations Research, 1(1), 35-42. Recuperado de http://ijmsoridi.com/index.php/ijmsor/article/view/75

Segura, E. (2016). Información, estabilidad y complejidad de aprendizaje en memorias asociativas. International Journal of Management Sciences and Operations Research, 1(1), 49-53. Recuperado de http://ijmsoridi.com/ index.php/ijmsor/article/view/77

Valencia, A., Suárez, R., Sánchez, A., Cardozo, E., Bonilla, M. y Buitrago, C. (2009). Gestión de la Contaminación Ambiental: Cuestión de corresponsabilidad. Revista de Ingeniería, 30. 90-99. 